\title{
Orthogonality Measurent of Ofdm Signal
}

\author{
Mahendra Sharma ${ }^{1}$, Santhosh Kumar Singh ${ }^{2}$ \\ ${ }^{1}$ Department of Information Technology, AMET University, Chennai, India \\ ${ }^{2}$ Department of Information Technology, Tagore College of Science \& Commerce, Mumbai, India
}

\begin{tabular}{l}
\hline \hline Article Info \\
\hline Article history: \\
Received Nov 17, 2017 \\
Revised Jan 23, 2018 \\
Accepted Feb 11, 2018 \\
\hline
\end{tabular}

Keywords:

Bit Error Rate (BER)

Inter-symbol Interference (ISI)

OFDM

Spectrum Analysis

\begin{abstract}
In recent days, Orthogonal Frequency Division Multiplexing is the technique to transmit and receive the signal without any overlapping of the signal. OFDM is also a multiplexing technique as well as modulation technique. It is a multi-carrier transmission technique in which single high data stream is divided into a number of lower rate streams that are transmitted simultaneous over some narrow sub channel. In general, OFDM avoids Inter-Symbol Interference (ISI), Inter-Carrier Interference (ICI) and fault transmissions between source and destination node. To measure the performance of the system by using the parameters like Bit Error rate (BER), Spectrum analysis and signal strength detection. Based on the parameters the best system can be identified.
\end{abstract}

Copyright (C) 2018 Institute of Advanced Engineering and Science. All rights reserved.

\section{Corresponding Author:}

Mahendra Sharma,

Department Information Technology,

AMET University, Chennai, India.

\section{INTRODUCTION}

Orthogonal Frequency Division Multiplexing (OFDM) is one of the emerging fields in wireless local area network that are also targeted for ad hoc network. OFDM can also be exploited in MANET to improve the energy and speed performance Mobile nodes in MANET are communicated directly through radio frequency range and wireless links. This process referred as peer o peer or single ad hoc network If the destination mobile node is out of range, then other nodes between source and destination act as router to transmit information between source and destination. This process is referred as multi-hop ad hoc networks. Single and Multi-hop ad hoc networks is illustrated in Figure 1.

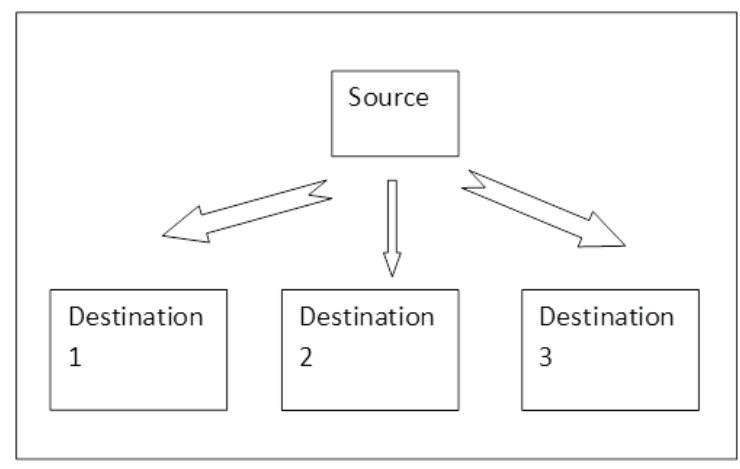

Figure 1. Single hop ad hoc network 


\section{BACKGROUND}

Mobile Ad Hoc Networks are self-configurable and infrastructure less networks consisting of mobile devices and routers which are able to support mobility and organize themselves arbitrarily [1]. It requires an extremely flexible technology for establishing communications between source and destination nodes. Design and performance analysis of MIMO-OFDM system using different antenna configurations is discussed in [2]. Hash based technique to identify the selfish node in Mobile Ad-hoc Network is explained in [3].

For the Modulation/De-modulation part of OFDM, Quadrature Amplitude Modulation (QAM) is frequently used for better Bit Error Rate (BER) and Signal to Noise Ratio (SNR). For the Channel Encoder/Channel Decoder of OFDM, Hamming codes are widely used for improving the error detecting probability [4]. Similarly, Single-path Delay Feedback (SDF) based IFFT/FFT structure is used for converting the time domain signal into frequency domain signal and vice versa [5]. Reconfigurable Metamaterial Structure at Millimeter Wave Frequency Range has been explained in [6]. In [7] presented the maximizing energy efficiency for consumption circuit power in downlink massive MIMO wireless networks The receiver side of OFDM consists of cyclic prefix removal for remove the added bits in transmitter side, Fast Fourier Transformation (FFT) for convert the time domain signal into frequency one, De-modulation (reverse process of modulation), Channel Decoder for decode the encoded word, and Source decoder to convert digital decoded binary information to analog one. Low power 128 point split radix fft for LTE application has been described in [8]-[9].

\section{THE PROBLEM}

OFDM technique faces many challenges like configuring different types of hardware architecture based on different types of application requirement. However, due to more complexity of hardware parts, single part adaptation is not sufficient to implement at appropriate time.

\section{THE PROPOSED SOLUTION}

The structure of OFDM transmitter and receiver is illustrated in Figure 2. The Encoder and Decoder of OFDM transmitter and receiver act as channel encoder and channel decoder which converts the source signals into set of binary information. One of the key techniques in which OFDM can be implemented on the sender side and receiver side for efficient communication is Fast Fourier Transformation (FFT). In general FFT is used to convert the time domain signals into frequency domain signals and vice versa.

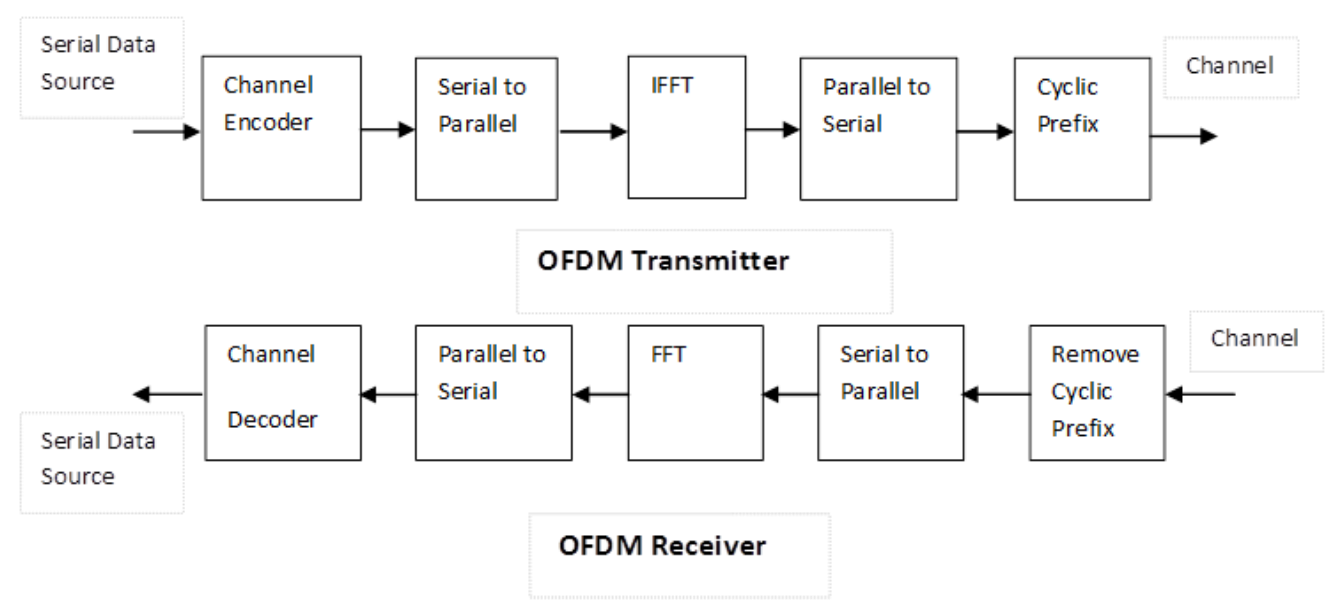

Figure 2. Architecture of OFDM System

Generally, convolutional encoder considered as a channel encoder of any wireless transmission techniques. The working principle of convolutional encoder is that the encoder performs a convolution of the input stream with impulse response. The process of convolutional encoder is further briefly described in [channel encoding]. Next to channel encoder, serial to parallel unit converts the serial information of channel encoder into parallel one for access all the inputs to IFFT unit at the same time. IFFT converts the time 
domain signals into frequency domain signals. Again parallel information from IFFT processor is converted into serial one with the help of parallel to serial block. Cyclic prefix of OFDM is used to eliminate the ISI and ICI by adding the guard interval or prefixing of bits into estimated binary signals [Cyclic prefix]. In receiver side of OFDM System, reverse operation is made to retrieve the original information signals. The cyclic prefix is removed in the receiver side of OFDM before the estimated signal is sent to FFT for demodulation. The presence of multipath fading channel and capability for parallel/pipelined processing of signal in OFDM make it a promising candidate for the next generation wide-band communications systems. The modulation and demodulation of OFDM System can be efficiently implemented with the help of IFFT and FFT transformation technique. The OFDM based communication systems need to have high performance in both power consumptions and throughput.

\section{RESULTS AND DISCUSSION}

The performance of the OFDM system and nature of the OFDM signal during the difficulty condition was analyzed using the Matlab simulation. Signal orthogonality, Bit error rate (BER), Spectrum analysis are predicted and evaluated. The results are shown in the Figure 3 and 4.

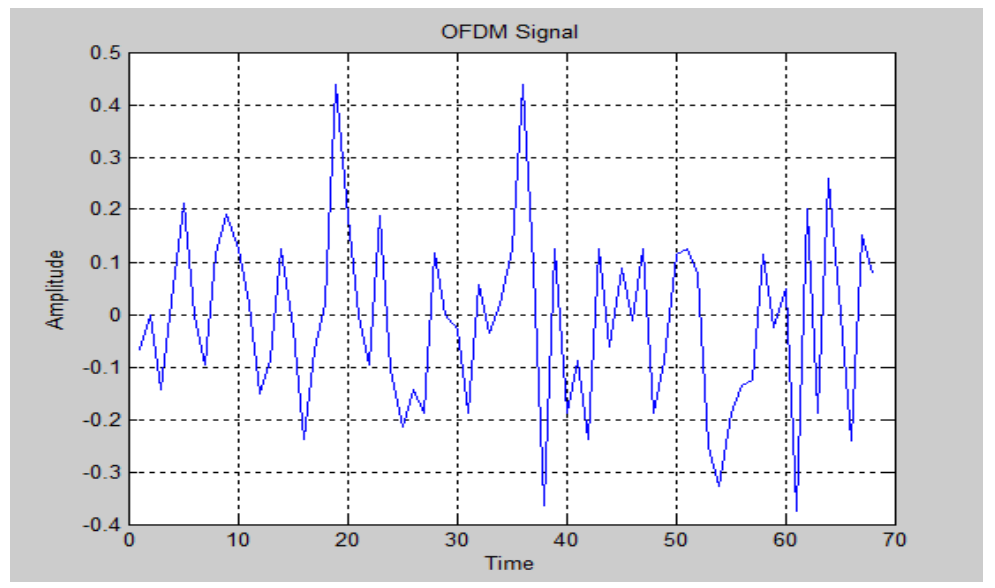

Figure 3. Spectrum Analyzes of OFDM Signal

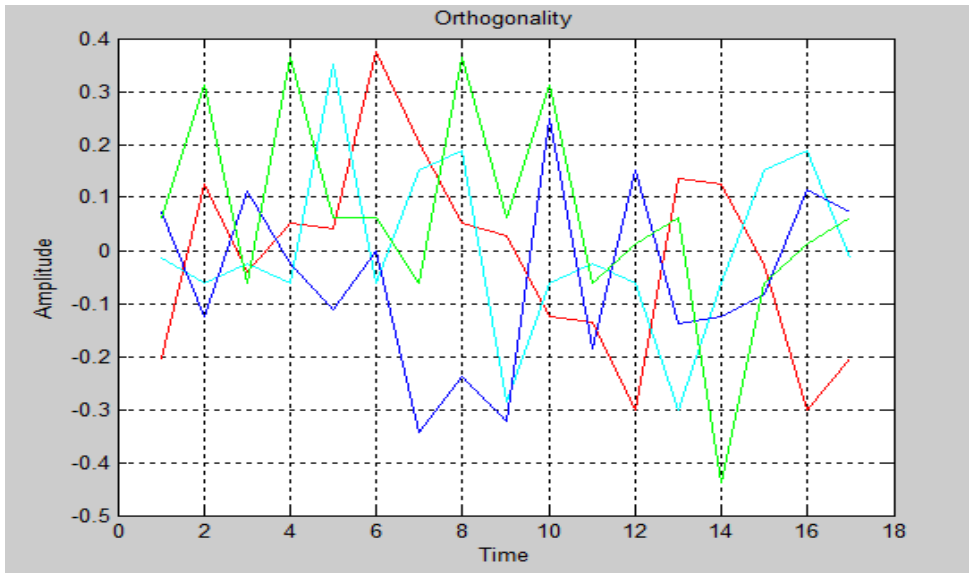

Figure 4. Orthogonality between different OFDM signals

\section{CONCLUSION}

The Performance of the OFDM signal was measured at different modulated signal. The signal orthogonality and spectrum was analyzed by using Matlab simulation tool. The output result clearly shows the performance of the individual signal. The technique gives the output signal with less number of 
distortions. So this method is used to transfer the signal from end to another over the long distance. It also provide non overlapping signal at the time of operation.

\section{REFERENCES}

[1] Yeung G, Takai M, Bagrodia R,Mehrnia A,Daneshrad B. Detailed OFDM Modeling in Network Simulation of Mobile Ad Hoc Networks. IEEE proceedings of the $18^{\text {th }}$ workshop on parallel and distributed simulation (PADS'04). 2004.

[2] Yu Q, Zheng J, Fu T, Wu K,and Zhang B. Asynchronous Cooperative Transmission Using Distributed Unitary Space-Frequency Coded OFDM in Mobile Ad Hoc Networks Published in IEEE future generation communication and networking (FGCN). 2007;(2): 291-296.

[3] Vennila G, and Arivazhagan D. Hash based Technique to Identify the Selfish Node in Mobile Ad-hoc Network. Indian Journal of Science and Technology. 2015; 8(14):1.

[4] Yang, Hongwei. A road to future broadband wireless access: MIMO-OFDM-based air interface. Communications Magazine.2005; 43,(1): 53-60.

[5] Agarwal A, and Mehta SN.Design and performance analysis of MIMO-OFDM system using different antenna configurations. IEEE International Conference on Electrical, Electronics, and Optimization Techniques (ICEEOT). 1373-1377.

[6] Esmail BA, Majid HA, Abidin ZZ, Dahlan SH, Rahim MK. Reconfigurable Metamaterial Structure at Millimeter Wave Frequency Range. International Journal of Electrical and Computer Engineering (IJECE). 2017;7(6).

[7] Salh A, Audah L, Shah NS, Hamzah SA. Maximizing Energy Efficiency for Consumption Circuit Power in Downlink Massive MIMO Wireless Networks. International Journal of Electrical and Computer Engineering (IJECE). 2017;7(6).

[8] Suman S, Sharma KG, Ghosh PK. $250 \mathrm{MHz}$ Multiphase Delay Locked Loop for Low Power Applications. International Journal of Electrical and Computer Engineering (IJECE). 2017;7(6).

[9] Shilpha G. Low power 128 point split radix fft for lte application. International Journal of MC square Scientific Research (IJMSR). 2014;6(1):66-74. 